\title{
HIV-1 integrase SUMOylation and viral replication
}

\author{
Alessia Zamborlini ${ }^{1,2^{*}}$, Audrey Coiffic ${ }^{1}$, Guillaume Beauclair ${ }^{1}$, Olivier Delelis ${ }^{3}$, Joris Paris ${ }^{1}$, Fabien Magne ${ }^{1,2}$, \\ Yashuiro Koh ${ }^{4}$, Marie-Lou Giron ${ }^{1}$, Joelle Tobaly-Tapiero', Stephane Emiliani ${ }^{5}$, Alan Engelman ${ }^{4}$, Hugues de The $^{1}$, \\ Ali Saib ${ }^{1,2}$
}

From Frontiers of Retrovirology 2011

Amsterdam, The Netherlands. 3-5 October 2011

\section{Background}

HIV-1 integrase catalyzes the integration of the reverse transcribed viral cDNA into the cellular genome, a key event of retroviral replication that is targeted by novel anti-HIV therapeutic agents. Numerous studies have contributed to understand of the molecular basis of integrase catalytic functions. Besides integration, HIV-1 integrase participates in other steps of viral replication such as reverse transcription and/or uncoating, PIC nuclear import and virion morphology. However, the underlying mechanisms are still not fully elucidated. Cellular and viral factors assist integrase in performing its multiple activities. Moreover, post-translational modifications (i.e. acetylation, ubiquitination and phosphorylation), which represent a common, rapid and generally reversible mechanism for fine tuning of protein activities, have also been shown to regulate integrase functions.

\section{Materials and methods and results}

By in vitro SUMOylation assay and purification on $\mathrm{Ni}$ NTA beads in denaturing conditions, we show that HIV1 integrase is covalently modified by the three SUMO paralogues. By mutating SUMO-acceptor lysine residues within phylogenetically conserved SUMOylation consensus motifs identified in silico, we demonstrate that they represent major sites of SUMO conjugation. We introduced the same changes in the integrase sequence of a plasmid encoding the viral genome, which was used to generate mutant viral particles, and we compare their infectivity to that of WT HIV-1. We find that viruses harboring SUMOylation-defective integrase mutants are less infectious that the WT counterpart. Real-time PCR analysis of viral cDNA synthesis reveals that cells infected with mutant viruses display similar content of traverse

${ }^{1}$ CNRS UMR7212, INSERM U944, Université Paris7 Diderot, Institut Universitaire d'Hématologie, Paris, 75010, France

Full list of author information is available at the end of the article transcripts, but a lower number of integrated proviruses, than WT HIV-1-infected cells. This integration defect at the integration step is not due to impairment of integrase binding to LEDGF/p7S, a key chromatin-tethering factor of HIV-1 pre-integration complex. Indeed, both WT and SUMOylation-defective integrase proteins coprecipitated with similar efficiency with LEDGF/p75. Finally, we establish that SUMOylation-defective integrase mutants retained WT catalytic activity as determined by Vprfusion protein complementation assay.

\section{Conclusions}

Here, we show that HIV-1 IN is modified by SUMO proteins and that phylogenetically conserved SUMOylation consensus motifs represent major SUMO-acceptor sites. Viruses harboring SUMOylation-site IN mutants displayed a replication defect that was mapped during the early stages of infection, before integration but after reverse transcription. Since SUMOylation-defective IN mutants retained WT catalytic activity as well as LEDGF-binding, we hypothesize that SUMOylation might regulate the affinity of IN for co-factors, contributing to efficient HIV-1 replication.

\section{Author details}

${ }^{1}$ CNRS UMR7212, INSERM U944, Université Paris7 Diderot, Institut

Universitaire d'Hématologie, Paris, 75010, France. ${ }^{2}$ Conservatoire National des Arts et Métiers, Department CASER, Paris, 75003, France. ${ }^{3}$ LBPA, CNRS

UMR8113, Ecole Normale Supérieure, Cachin, 94235, France. ${ }^{4}$ Dana-Farber Cancer Institute, Department of Cancer Immunology \& AIDS, Boston, MA, 02115, USA. ${ }^{5}$ INSERM U1016, CNRS JMR8104, Université Paris Descartes, Cochin Institut, Paris, 75014, France.

Published: 3 October 2011

doi:10.1186/1742-4690-8-S2-04

Cite this article as: Zamborlini et al:: HIV-1 integrase SUMOylation and viral replication. Retrovirology 2011 8(Suppl 2):O4. 